\section{E-093 ACUTE ISCHEMIC STROKE FROM FIBROCARTILAGINOUS EMBOLISM TO THE BASILAR ARTERY}

J Tatum*, A Marshall, T Madaelil. Texas Cerebrovascular and Stroke Institute, Plano, TX

10.1136/neurintsurg-2021-SNIS. 188

Purpose Fibrocartilaginous embolization from the nucleus pulposus is a rare cause of spinal cord ischemia, however, only one case of fibrocartilaginous cerebral embolism (FCE) has been previously described. ${ }^{1}$ To our knowledge this is the first reported case of mechanical embolectomy (ME) in a patient with fibrocartilaginous cerebral embolism.

Case A 51 y/o woman with a history of left shoulder pain and steroid injections presented with acute onset of dysarthria, nystagmus, dysmetria and diplopia with an NIHSS of 6. CTA revealed a sub-total occlusive filling defect at the C5/C6 level in the dominant left VA V2 segment, a partial filling defect in the mid BA and occlusion of the distal BA extending into the left P1 segment. The patient met criteria for mechanical embolectomy (ME). Standard aspiration thrombectomy was performed with a Catalyst 6 aspiration catheter through a Cerebase sheath. Three total aspiration thrombectomy passes were performed with ultimate reperfusion of the basilar artery. Distal migration of an embolus into the left P2 segment, prompted a fourth and final combined stent retriever $(3 \times 20$ $\mathrm{mm}$ Trevo) and aspiration pass, with unchanged distal occlusion. The patient made an excellent recovery.

Discussion In review of the CTA of the neck, there is a prominent lateral disc protrusion at C5-C6 with a filling defect in the adjacent medial VA. An MRI c-spine confirmed a lateral disc protrusion at this level. In combination with a lateral disc osteophyte, this may have led to medial disruption of the VA and subsequent herniation of fibrocartilage into the left VA leading to the patient's embolism. A concordant fine linear filling defect was seen at C5-C6 on cervical angiography. Only one case of FCE resulting in cerebral ischemia exists in the literature. In that case, a 17-year-old female developed a right cerebral infarct and died from complications of her stroke. Autopsy confirmed FCE in the cerebral and coronary vessels suggesting a central source of embolism. In contrast, in our case, a lateral disc extrusion into the left VA through a defect created by the disc and/or disc osteophyte is postulated as the etiology of embolism. In this unusual case of FCE, aspiration thrombectomy removed this foreign material from the BA. Distal clot was not secured by stent-retriever. We believe the fibrous nature of the embolus could explain this observation, similar to experience in calcific emboli as reported by Dobrocky et al. ${ }^{2}$

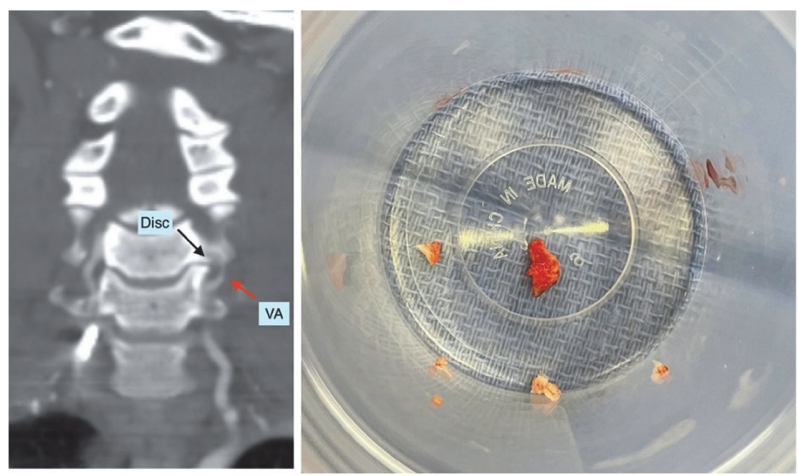

Abstract E-093 Figure 1
REFERENCES

1. Stroke. 1993 May; 24(5):738-40.

2. JNIS. 2017 April;10(4):345-350

Disclosures J. Tatum: None. A. Marshall: None. T. Madaelil: None.

\section{E-094 CORE-LAB ADJUDICATED OUTCOMES OF SINGLE-STENT ASSISTED COILING FOR WIDE-NECK BIFURCATION ANEURYSMS: A MULTICENTER STUDY}

${ }^{1} \mathrm{D}$ Bageac*, ${ }^{2} \mathrm{~S}$ Gomez-Paz, ${ }^{3} \mathrm{~J}$ Vranic, ${ }^{1} \mathrm{~B}$ Gershon, ${ }^{3} \mathrm{~W}$ Munoz Miranda, ${ }^{3} \mathrm{C}$ Stapleton, ${ }^{4} \mathrm{~K}$ Fargen, ${ }^{2} \mathrm{~A}$ Thomas, ${ }^{3} \mathrm{~A}$ Patel, ${ }^{1} \mathrm{R}$ De Leacy. ${ }^{1}$ The Mount Sinai Hospital, New York, NY; ${ }^{2}$ Beth Israel Deaconess Medical Center, Boston, MA; ${ }^{3}$ Massachusetts General Hospital, Boston, MA; ${ }^{4}$ Wake Forest Baptist Health, Winston Salem, NC

\subsection{6/neurintsurg-2021-SNIS.189}

Background/Purpose Single stent-assisted coiling is one option in the endovascular treatment of wide neck bifurcation aneurysms. In comparison to double-stent configurations, single-stent assisted coiling is less procedurally complex, and thus may offer an improved safety profile. This may come at the expense of efficacy, because the aneurysm neck is only partially reconstructed. Core-lab adjudicated real world evidence regarding the safety and efficacy of single-stent assisted coiling for WNBAs is lacking. We present the results of a multicenter core-lab adjudicated study of outcomes following single-stent assisted coiling of WNBAs.

Methods Cases of single stent-assisted coiling of WNBAs occurring at 3 large academic centers were identified. WNBAs were defined as bifurcation aneurysms with neck diameter $>$ $4 \mathrm{~mm}$ or dome-to-neck ratio $<2$. Baseline clinical characteristics and clinical outcomes were determined by retrospective review of patient records. Aneurysm characteristics and procedural details were determined by prospective review of patient imaging accompanied by retrospective review of operative reports. Immediate and final follow-up occlusion scores and the development of in-stent stenosis were determined by an independent core lab.

Results Inclusion criteria were met by 126 patients harboring 129 WNBAs treated by single-stent assisted coiling. Previously treated aneurysms accounted for $23.3 \%$ of cases, $17.9 \%$ had a remote history of rupture, and one $(0.7 \%)$ was acutely ruptured. Aneurysms were located as follows: anterior communicating artery (54.3\%), middle cerebral artery bifurcation $(21.0 \%)$, basilar apex $(16.3 \%)$, internal carotid artery terminus $(4.7 \%)$, and distal anterior cerebral artery bifurcation (3.1\%). With regard to size, $23.3 \%$ of aneurysms were $<5 \mathrm{~mm}$ in diameter, $71.3 \%$ were $\geq 5 \mathrm{~mm}$ and $<13 \mathrm{~mm}$, and the remaining $5.4 \%$ were $\geq 13 \mathrm{~mm}$. Stents used included the

Neuroform Atlas (40.3\%), LVIS Jr. (40.3\%), Neuroform EZ (14.0\%), and LVIS BLUE (5.4\%). A microcatheter jailing technique was employed in $62.0 \%$ of cases, and a trans-cell microcatheter technique was used in the remaining 38.0\%.

The median follow-up interval was 14.9 months (IQR 7.0 - 19.6). Over the course of the follow-up period, $10.8 \%$ of aneurysms (14/129) required repeat endovascular treatment due to recanalization. Preliminary analysis of noncore-lab adjudicated angiographic outcomes at last available follow-up demonstrated the following occlusion rates: 75.2\% mRR I, 15.5\% mRR II, 3.9\% mRR IIIa, 5.4\% mRR 